\title{
Ensuring Security in Emergency through SMS Alert System
}

\author{
A.Sankari \\ Prist University \\ Thanjavur, India
}

\author{
K.Umasankar \\ Prist University \\ Thanjavur, India
}

\begin{abstract}
Short Message Services are increasingly being relied upon to disseminate critical information during emergencies. In recent days accident happens very common due to heavy traffic and increase in vehicle level and bad drivers, hence it requires a sof tware to inform the service centre for instant help to save life of the people. Sending the nearest emergency service and/or police officers needed for reporting the accident can be quite tedious. The idea of this work is to reduce the time required by the emergency personnel to reach the accident area. This can be done if the information about an accident reaches the emergency services in time and accurately. The project eliminates any communication between the victim and the personnel which leads to confusion. This is done by finding the accurate position of the location by making use of the GPS services available in cell phones when the victim sends a message to the emergency number(such as 108). The project aims at reducing the severe loss due to injury and fatality rate in accidents to a great extent.
\end{abstract}

Keywords: GPS services, Short Message Services

\section{INTRODUCTION}

SMS allows individuals to transmit short, alphanumeric communications for a wide variety of applications. Whether to coordinate meetings, giving alert notification, offer reminders of an event or even vote for a contestant on a television game show, this discreet form of communication is now the dominant service offered by cellular networks. In fact, in the United States alone, over five billion text messages are delivered each month. While many of the applications of this service can be considered noncritical, the use of text messaging during emergency events has proven to be far more utilitarian. Premium-rated messages are also used in Donors Message Service to collect money for charities and foundations. DMS was first launched at April 1, 2004, and is very popular in the Czech Republic. For example, the Czech people sent over 1.5 million messages to help South Asia recover from the 2004 Indian Ocean Earthquake.

With millions of people attempting to contact friends and family on September 11th 2001, telecommunications providers witnessed tremendous spikes in cellular voice service usage. Verizon Wireless, for example, reported voice traffic rate increases of up to 100 percent above typical levels; Cingular Wireless recorded an increase of up to 1,000 percent on calls destined for the Washington D.C. area. While these networks are engineered to handle elevated amounts of traffic, the sheer number of calls was far greater than capacity for voice communications in the affected areas. However, with voice based phone services being almost entirely unavailable, SMS messages were still successfully received in even the most congested regions because the control channels responsible for their delivery remained available. Similar are the stories from the Gulf Coast during Hurricanes Katrina and Rita. With a large number of cellular towers damaged or disabled by the storms, text messaging allowed the lines of communication to remain open for many individuals in need, in spite of their inability to complete voice calls in areas where the equipment was not damaged and power was available.

\section{PROPOSED SYSTEM}

The proposed system requires the affected person to just send a message to the service center from their mobile. The message is sent through a software installed in mobile which doesn't require the user to type any text. Then the message will be received by the service center using GSM modem. The proposed system configuration is shown in Fig. 1. The latitude and longitude value of the place from which the message is sent is received in the server. The geo coding used in the server side now converts these values to precise location. The service center can now view the accident location on a map with the details about the nearest emergency services. Then they inform the type of the accident and appropriate location to the nearby service (police $\&$ fire). This will reduce the time required for the police and the emergency personnel to reach the accident location. The idea makes the location identification automatic. This helps in identifying the accident spot precisely and quickly. Hence it reduces the time required for the emergency services to reach the spot.

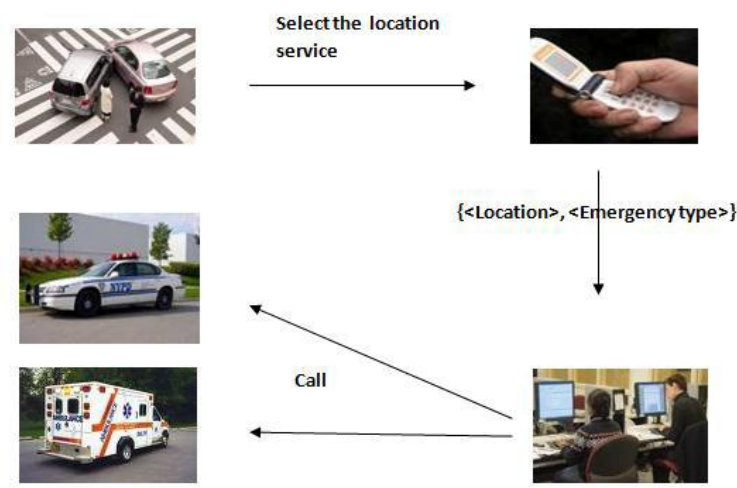

Fig 1 : Block diagram of proposed system

The technology used can be developed with the current equipment like GSM modem and Geo Code technique, and has the technical capacity to hold the data required by the new system.
1. This technology supports the modern trends of technology.
2. Easily accessible, more secure technologies.

Technical feasibility on the existing system and to what extend it can support the proposed addition. MVC and geo code is latest technique used by many smart phones now a days. We can add new modules easily without affecting the 
Core Program. Most of parts are running in the server using the concept of stored procedures. Ensuring Security Intimations in emergency through SMS Alert

System implements and satisfied the technical feasibility level.

\subsection{Operational Feasibility}

This proposed system can easily implemented, as this is based on JSP coding (JAVA) \& HTML . The database created is with MySql server which is more secure and easy to handle. The resources that are required to implement/install these are available. The personal of the organization already has enough exposure to computers. So the project is operationally feasible. It uses Mysql server for easy handling and GSM for better SMS receiving rates. It is easy to operate by non-technical users also.

\subsection{Economical Feasibility}

Economic analysis is the most frequently used method for evaluating the effectiveness of a new system. More commonly known cost/benefit analysis, the procedure is to determine the benefits and savings that are expected from a candidate system and compare them with costs. If benefits outweigh costs, then the decision is made to design and implement the system. An entrepreneur must accurately weigh the cost versus benefits before taking an action. This system is more economically feasible which assess the brain capacity with quick \& online test. So it is economically a good project. This emergency alert project used open source software for development so it is freely available and free of cost. And today most users have android mobile phones. There is no extra hardware cost for all. The advantages of the proposed system are

1. It eliminates any conversation between the victim and the service center. Hence it reduces any confusion and possibility of wrong information.

2. Software requirement is very less, hence it can be implemented easily. It does not require any specialized hardware to be installed.

3. There is no need of regular update. Even if there is any update it can be downloaded easily and does not require any cost.

4. Even the affected person can inform about the accident using the proposed system. Since it does not require typing any text or making a call it becomes simple for the victim.

5. Since messages can be sent even with low signals, it reduces any technical problem. Messages can be sent from places where making calls is not possible. Hence possibility of message reaching the service center is higher when compared to calls.

6. It is cheaper to use

\section{CELLULAR NETWORK ARCHITECTURE}

In this section, we provide a technical overview of message delivery to understand how such cellular networks deliver text messages and a high-level description of how third-party vendors try to use these systems to deliver alert messages during emergency. We specifically examine GSM networks [3] in these discussions as they represent the most widely deployed cellular technology in the world; however, it should be noted that message delivery for other technologies such as CDMA, IDEN, and TDMA are very similar and are therefore subject to similar problems.

\subsection{Sending a Message}

There are a number of ways in which text messages can be injected into a GSM or CDMA network. While most users are only familiar with sending a text message from their phone, known as Mobile Originated SMS (MO-SMS), service providers offer an expanding set of interfaces through which messages can be sent. From the Internet, for instance, it is possible to send text messages to mobile devices through a number of webpages, e-mail, and even instant messaging software. Third parties can also access the network using socalled SMS Aggregators. These servers, which can be connected directly to the phone network or communicate via the Internet, are typically used to send "bulk" or large quantities of text messages. Aggregators typically inject messages on behalf of other companies and charge their clients for the service. Finally, most providers have established relationships between each other to allow for messages sent from one network to be delivered in the other. Fig. 2 shows these three high-level strategies. After entering a provider's network, messages are sent to the Short Messaging Service Center (SMSC). SMSCs perform operations similar to e-mail handling servers in the Internet, and store and forward messages to their appropriate destinations. Because messages can be injected into the network from so many external sources, SMSCs typically perform aggressive spam filtering on all incoming messages. All messages passing this filtering are then converted and copied into the necessary SMS message format and encoding and then placed into a queue to be forwarded to their final destination.

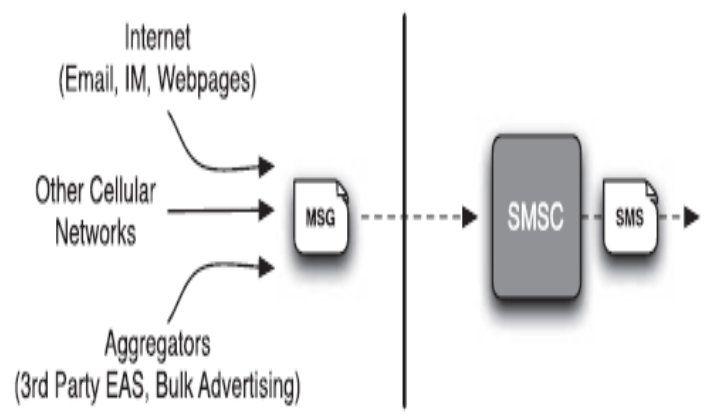

External Networks

Provider Network

Fig. 2 : Processing of Text message

\subsection{Finding a Device}

Delivering messages in a cellular network is a much greater challenge than in the traditional Internet. Chief in this difficulty is that users in a cellular network tend to be mobile, 
so it is not possible to assume that users will be located where we last found them. Moreover, the information about a user's specific location is typically limited. For instance, if a mobile device is not currently exchanging messages with a base station, the network may only know a client's location at a very coarse level (i.e., the mobile device may be known to be in a specific city, but no finer grained location information would be known). Accordingly, the SMSC needs to first find the general location for a message's intended client before anything else can be done.

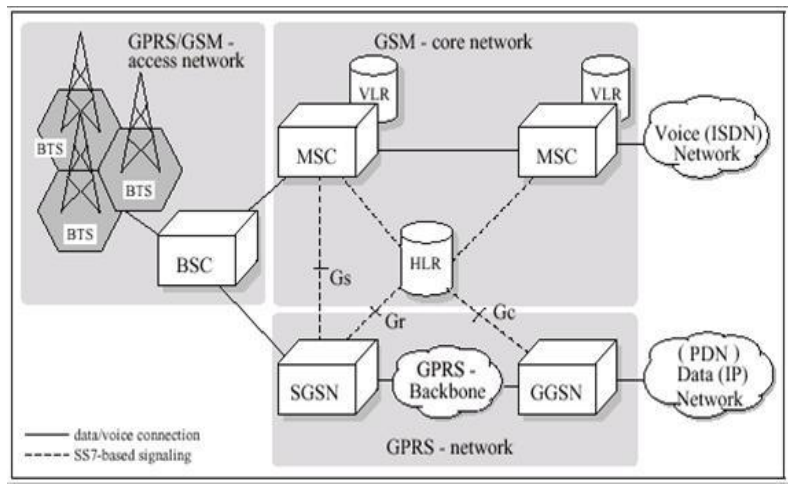

Fig. 3 : Network Architecture

A server known as the Home Location Register (HLR) assists in this task. This database acts as the permanent repository for a user's account information (i.e., subscribed services, call forwarding information, etc.). When a request to locate a user is received, the HLR determines whether or not that device is currently turned on. If a mobile device is currently powered off, the HLR instructs the SMSC to store the text message and attempt to deliver it at another time. Otherwise, the HLR tells the SMSC the address of the Mobile Switching Center (MSC) currently serving the desired device. Having received this location information, the SMSC then forwards the text message on to the appropriate MSC.

\subsection{Wireless Delivery}

As mentioned earlier, even the MSC may not know more information about a targeted device's location. In order to determine whether or not the current base station serving this device is known, the MSC queries the Visitor Location Register (VLR), which temporarily stores information about clients while they are being served by the MSC. In most cases, this information is not known, and so the MSC must begin the extensive and expensive process of locating the mobile device. The MSC completes this task by generating and forwarding paging requests to all of its associated base stations, which may number in the hundreds. This process is identical to locate a mobile device for delivery of a voice call. Upon receiving a paging request from the MSC, a base station attempts to determine whether or not the targeted device is nearby. To achieve this, the base station attempts to use a series of Control Channels to establish a connection with the user. First, the base station broadcasts a paging request over the Paging Channel (PCH) and then waits for a response. If the device is nearby and hears this request, it responds to the base station via the Random Access Channel (RACH) to alert the network of its readiness to receive information. When this response is received, the network uses the Access Grant Channel (AGCH) to tell the device to listen to a specific Standalone Dedicated Control Channel (SDCCH) for further exchanges. Using this SDCCH, the network is able to authenticate the client, perform a number of maintenance routines and deliver the text message. By limiting the operations necessary to deliver a text message to the control channels used for call setup, such messages can be delivered when all call circuits, known as Traffic Channels (TCHs) are busy. When the attempt to deliver the message between the targeted device and the base station is complete, the device either confirms the success or failure of delivery. This status information is carried back through the network to the SMSC. If the message was successfully delivered, the SMSC deletes it. Otherwise, the SMSC stores the message until a later period, at which time the network reattempts delivery. Fig. 2 offers an overview of this entire process.

\subsection{Third-Party Provider Solutions}

In the past few years, a significant number of thirdparties offering to deliver alert messages (and other information services) via text messaging services. The architecture of these systems is relatively simple. Whether activated through a web interface, directly from a phone, or as software running on a campus administrator's computer, these services act as SMS aggregators and inject text messages into the network. In the event of an emergency message is sent to the service center from the victim or passerby mobile

\subsection{Short Message Service}

Short Message Service (SMS) is a text messaging service component of phone, web, or mobile communication systems, using standardized communications protocols that allow the exchange of short text messages between fixed line or mobile phone devices. SMS text messaging is the most widely used data application in the world, with 3.6 billion active users, or $78 \%$ of all mobile phone subscribers. The term SMS is used as a synonym for all types of short text messaging as well as the user activity itself in many parts of the world. SMS is also being used as a form of direct marketing known as SMS marketing. Simple user generated text message services - include news, sport, financial, language and location based services, as well as many early examples of mobile commerce such as stocks and share prices, mobile banking facilities and leisure booking services. SMS as used on modern handsets originated from radio telegraphy in radio memo pagers using standardized phone protocols and later defined as part of the Global System for Mobile Communications (GSM) series of standards in $1985^{1}$ as a means of sending messages of up to 160 characters, to and from GSM mobile handsets. Since then, support for the service has expanded to include other mobile technologies such as ANSI CDMA networks and Digital AMPS, as well as satellite and Landline networks. Most SMS messages are mobile-to-mobile text messages though the standard supports other types of broadcast messaging as well.

\subsection{GSM Technology}

GSM is a cellular network, which means that cellphones connect to it by searching for cells in the immediate vicinity. There are 5 different cell sizes in a GSM network. The coverage area of each cell varies according to the implementation environment. Indoor coverage is also supported by GSM. GSM uses several cryptographic algorithms for security. A convenient facility of the GSM network is the short message service. 


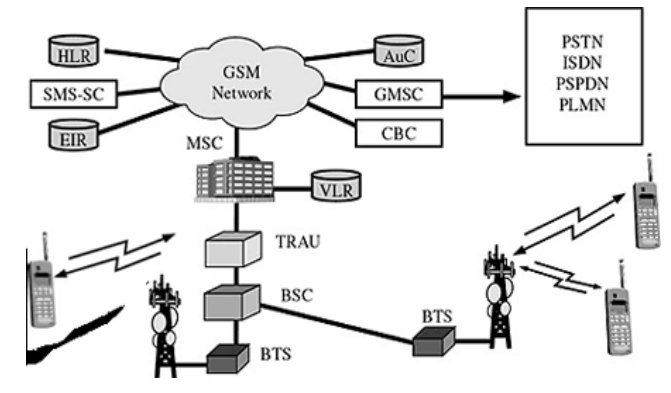

Fig. 4 : GSM Network along with SMSC

The Short Message Service - Point to Point (SMS-PP) was originally defined in GSM recommendation 03.40 , which is now maintained in 3GPP as TS 23.040. GSM 03.41 (now 3GPP TS 23.041) defines the Short Message Service - Cell Broadcast (SMS-CB), which allows messages (advertising, public information, etc.) to be broadcast to all mobile users in a specified geographical area. Messages are sent to a Short message service center (SMSC) which provides a "store and forward" mechanism. It attempts to send messages to the SMSC's recipients. If the subscriber's mobile unit is powered off or has left the coverage area, the message is stored and offered back to the subscriber when the mobile is powered on or has reentered the coverage area of the network. This function ensures that the message will be received.

Both mobile terminated (MT, for messages sent to a mobile handset) and mobile originating (MO, for those sent from the mobile handset) operations are supported. In Message delivery, delay or complete loss of a message is uncommon, typically affecting less than $5 \%$ of messages.

\subsection{Data Base System}

Database is required to store the information received through the message using GSM modem. The information can be then taken for further processing. The data stored in the database are the mobile number, the type of accident, the latitude value, the longitude value, the date and the time when the message was sent. First a connection between the server and the database is established using JDBC. Then the transfer of data takes place.
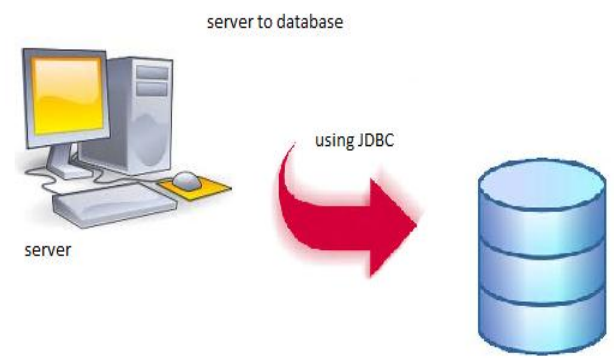

database

Fig. 5. Database Transfer system

JDBC allows multiple implementations to exist and be used by the same application. The API provides a mechanism for dynamically loading the correct Java packages and registering them with the JDBC Driver Manager. The Driver Manager is used as a connection factory for creating JDBC connections.

JDBC connections support creating and executing statements. Additionally, stored procedures may be invoked through a JDBC connection. It finds the exact location of the accident. The server then retrieves the contact details of the nearest emergency services from the databases and then inform them about the accident and its location.

\section{TESTING RESULTS}

This proposed system is tested from various mobile and different places by sending SMS to a designated emergency number. The screenshots of the results are furnished below.

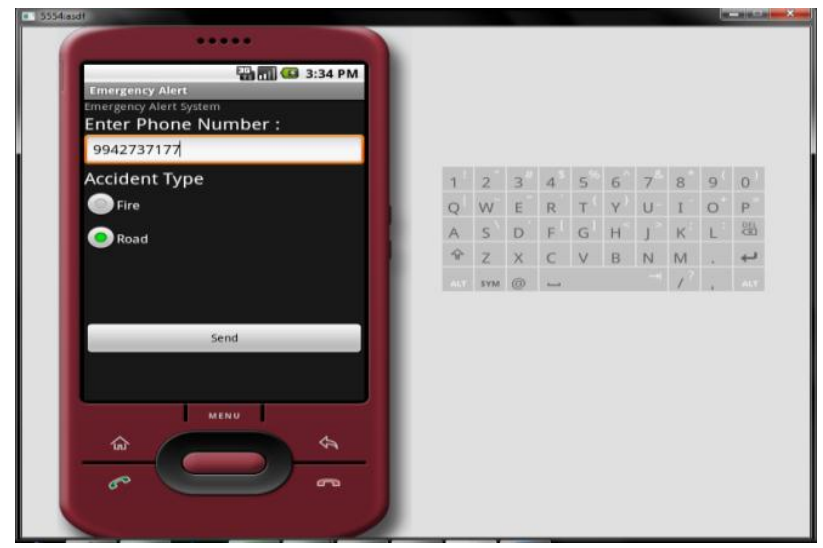

Fig. 6 : Software installed in Mobile phone for sending message

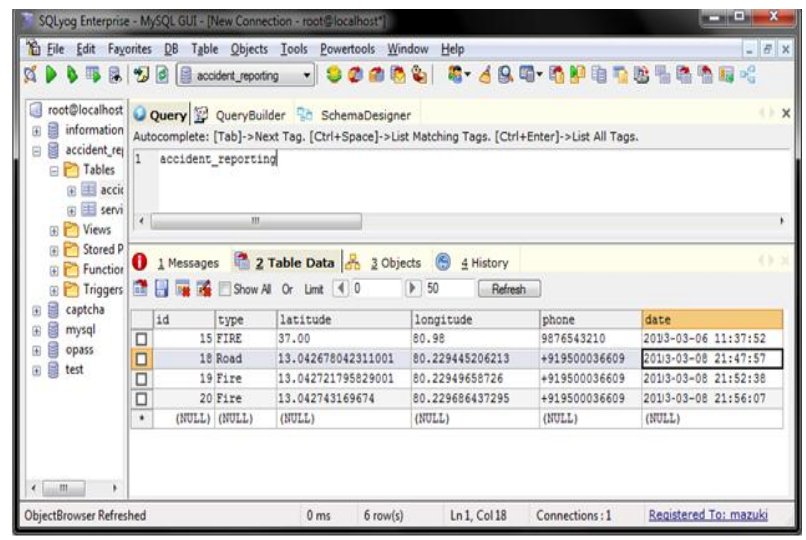

Fig. 7 : Details Stored in Database

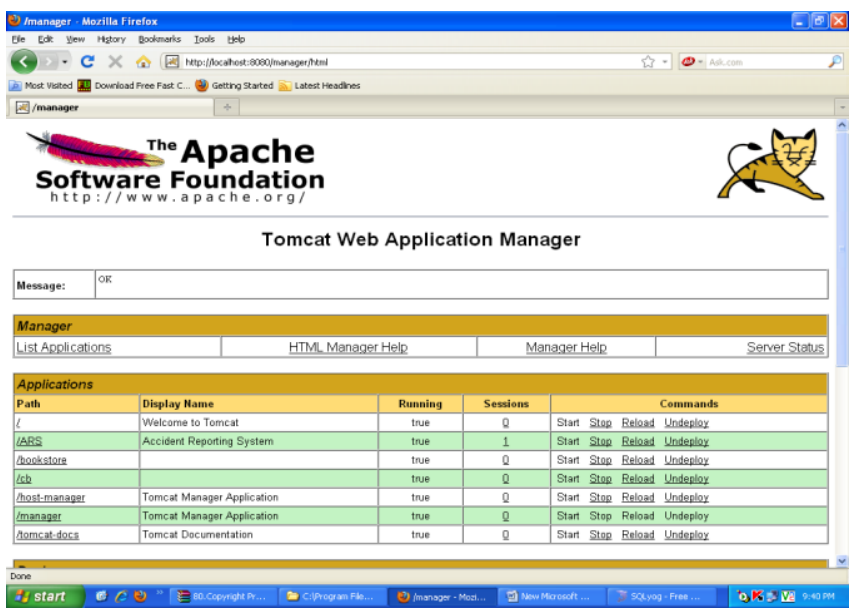

Fig. 8 : Running Apache server 




Accident Reporting System
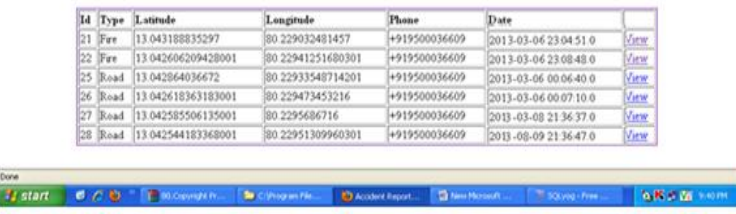

Fig. 9 : Precise location using geocoding technique

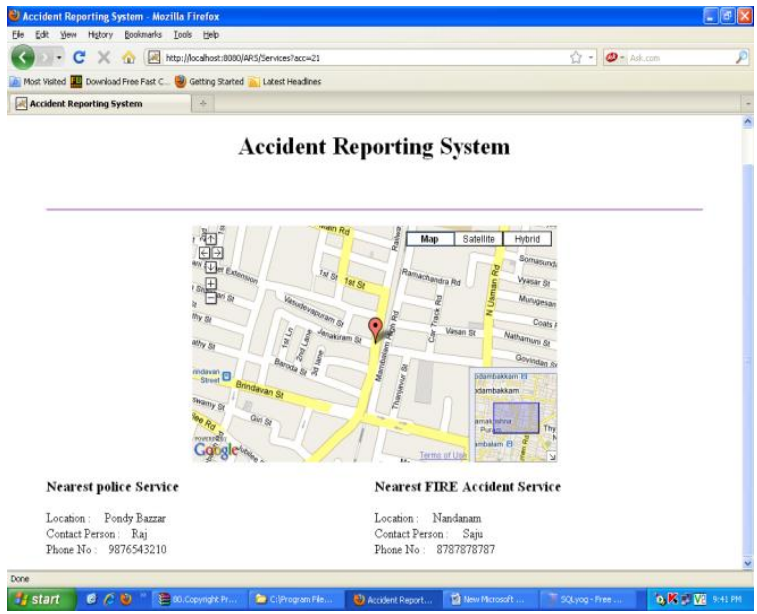

Fig. 10 : Viewing location in the map

\section{CONCLUSION}

The proposed SMS alert system is aimed to reduce the time required by the rescue team to reach the accident area. The message is sent through a software installed in mobile. Even the affected person can inform about the accident using the proposed system. It uses GPS to send the latitude and longtitude value of a place from which the messages is sent. The server receives the message using GSM Modem and uses reverse geocoding to convert latitude and longtitude value to exact location where the accident took place. The acknowledgement is sent from the service centre to the victim which reduces the panic in the person. There is neither any maintenance nor any service required for this system. Since messages can be sent even with low signals, it reduces any technical problem.

\section{REFERECES}

[1] Daniel Adkins, Karthik Lakshminarayanan, Adrian Perrig, and Ion Stoica. Taming IP packet flooding attacks. In Proceedings of Workshop on Hot Topics in Networks (HotNets-II), November 2003

[2] Patrick Traynor, "Characterizing the Security implications of Third-Party Emergency Alert Systems over Cellular Text Messaging Services", IEEE / Trans. on Mobile Computing, vol. 11, no. 6, June 2012

[3] "Technical Realization of the Short Message Service (SMS),"Technical Report 3GPP TS 03.40 v7.5.0. 3rd Generation Partner-ship Project, 2002.

[4] "Analysis of the Short Message Service (SMS) and Cell Broadcast Service (CBS) for Emergency Messaging Applications; Emergency Messaging; SMS and CBS," Technical Report ETSI TR 102444 V1.1.1. European Telecomm. Standards Inst., 2006.

[5] P. Bosch, L. Samuel, S. Mullender, P. Polakos, G. Rittenhouse, G., "Flat Cellular (UMTS) Networks," Wireless Communications and Networking Conference, 2007.WCNC 2007. IEEE

[6] R. Doverspike and A. Gerber, "Traffic Types and Growth in Backbone Networks," in Proc. of OFC/NFOEC, Invited Paper, 2011

[7] Jochen Schiller, Mobile Communications, II Edition, Addison Wesley

[8] Lu, W., Giordano, S. (2001) 'Challenges in Mobile Ad Hoc Networking,' collection of articles, IEEE Communications Magazine, 39(6).

[9] Campbell, A., Gomez, J., Kim, S., Valko, A., Wan, C. Y., Turanyi, Z. (2000) 'Design, implementation and evaluation of Cellular IP,' IEEE Personal Communications, 7(4).

[10] Location management in cellular networks. In I. Stojmenovic (Ed.), Handbook of wireless networks and mobile computing (pp. 24-49). New York: John Wiley $\&$ Sons.

[11] Lin, Y.-D., \& Hsu, Y.-C. (2000). Multihop cellular: A new architecture for wireless communications. Proceedings of IEEE INFOCOM.

[12] T.S.Ueng, Z.D.Tsai, J.C.Chang (2007) "SMS alert system AT NSRRC",Taiwan. Proceedings of PAC07, New Mexico, USA.

[13] Nilesh Agarwal, Leena Chandran-Wadia, Varsha Apte "Capacity Analysis of the GSM Short Message Service" Indian Institute of Technology Bombay. 